\title{
EFFICIENT TRAINING OF RBF NETWORKS FOR CLASSIFICATION
}

\author{
IAN T. NABNEY \\ Neural Computing Research Group, \\ Aston University, Birmingham, B4 7ET, UK \\ i.t.nabney@aston.ac.uk
}

Received 10 February 2003

Revised 29 Octcber 2003

Accepted 28 April 2003

\begin{abstract}
Radial Basis Function networks with linear outputs are often used in regression problems because they can be substantially faster to train than Multi-layer Perceptrons. For classification problems, the use of linear outputs is less appropriate as the outputs are not guaranteed to represent probabilities. We show how RBFs with logistic and softmax outputs can be trained efficiently using the Fisher scoring algorithm. This approach can be used with any model which consists of a generalised linear output function applied to a model which is linear in its parameters. We compare this approach with standard non-linear optimisation algorithms on a number of datasets.
\end{abstract}

Keywords: Author to provide.

\section{Introduction}

Radial Basis Function (RBF) networks with linear outputs are often used in regression problems because they can be substantially faster to train than Multi-layer Perceptrons (MLP). This is because it is possible to choose suitable parameters for the basis function parameters by an unsupervised technique (such as selecting a subset of the data for centres, using a clustering algorithm such as $K$-means, or training a mixture model with EM) so that the hidden unit activations model the unconditional input data density $p(\mathbf{x})$. With the hidden unit parameters fixed, and a sum of squared error function, the optimisation of the outputs weights is a quadratic problem that can be solved using the methods from numerical linear algebra.

In classification problems, rather than directly outputting a classification it is advantageous to estimate posterior probabilities $p\left(C_{k} \mid \mathbf{x}\right)$, since this allows us to compensate for different prior probabilities, combine the outputs of several networks, make minimum risk classifications under different cost functions and to set rejection thresholds. ${ }^{1}$ For classification problems, the use of linear outputs is less appropriate as then the network outputs are not guaranteed to represent probabilities. With MLPs it is common practice to use logistic (for two class) and softmax (for multiple classes) output nodes and appropriate cross-entropy error function so as to ensure that the outputs sum to one and all lie in the interval $[0,1]$. This does not add significantly to the time taken to train an MLP since even with linear outputs, general purpose optimisation routines must be used.

However, an RBF with logistic or softmax outputs no longer has a quadratic error surface for the output layer. If general purpose optimisation algorithms are used, much of the speed advantage over MLPs is lost. In this paper we show how RBFs with logistic and softmax outputs can be trained efficiently using algorithms derived from Generalised Linear Models. We compare these models with standard RBF's on both synthetic and real datasets. 


\section{Training Generalised Linear Models}

A generalised linear model is one where a non-linear output function is applied to a standard linear regression model. In this section, we first discuss the choice of the output function and then a particularly efficient parameter estimation algorithm.

\subsection{Generalising linear regression}

This brief outline of generalised linear models is based on that in McCulagh and Nelder. ${ }^{2}$ In linear regression theory, it is assumed that the errors follow a normal distribution with constant variance $\sigma^{2}$. The output of the model represents the mean conditioned on the input vector $\mathbf{x}$ :

$$
\mu=\mathbf{x} \boldsymbol{\beta} .
$$

In a generalised linear model we replace the normal distribution for the target random variable $Y$ by a distribution from the exponential family, which has the form:

$P_{Y}(y, \theta, \phi)=\exp \{(\theta y-b(\theta)) / a(\phi)+c(y, \phi)\}$,

where $\theta$ is the "natural parameter" and $\phi$ is the "dispersion parameter". It is easy to show that the mean $\mu$ of $P_{Y}$ is a function of $\theta: \mu=b^{\prime}(\theta)$. For the normal distribution, $\theta=\mu$ and $\phi=\sigma^{2}$. The generalised linear model has the form

$$
\eta=\mathbf{x} \boldsymbol{\beta} \text { and } \theta=f(\eta),
$$

where $f$ is the link function. If $\eta=\theta$ (i.e.. $f$ is the identity), then the output of the generalised linear model is the natural parameter of the noise model, and $f=b^{\prime}$ is the canonical link. Both of the generalised linear models we consider in this paper use a canonical link function.

The normal distribution is not an appropriate error model for classification problems, for which the output variable is discrete and not continuous. For a two-class problem, we use a Bernoulli distribution

$$
\begin{aligned}
P(y) & =\pi^{y}(1-\pi)^{1-y} \\
& =\exp \left\{\eta y-\ln \left(1+e^{\eta}\right)\right\},
\end{aligned}
$$

where $\pi$, the probability of "success" is the mean, and $\eta=\ln (\pi /(1-\pi))$. This corresponds to using a logistic function $\pi=1 / 1+\exp (-\eta)$ at the output of the generalised linear model.
For an $m$-class problem, we use the multinomial distribution on $m$ variables:

$$
\begin{aligned}
& P\left(y_{1}, y_{2}, \ldots, y_{m}\right) \\
& \quad=\frac{M !}{\left(y_{1} !\right)\left(y_{2} !\right) \cdots\left(y_{m} !\right)} p_{1}^{y_{1}} p_{2}^{y_{2}} \cdots p_{m}^{y_{m}},
\end{aligned}
$$

where $p_{i}$ is the probability of the $i$ th class and $M=\sum_{i=1}^{m} y_{i}$ is generally taken to be equal to one. This implies that the class is represented with a oneof- $m$ encoding, so that each vector contains zeros everywhere except for the correct coordinate which is 1 . The model has $m$ outputs and the canonical link function is the familiar softmax function:

$$
p_{i}=\frac{e^{\eta_{i}}}{\sum_{j=1}^{m} e^{\eta_{j}}},
$$

where $\eta_{1}, \ldots, \eta_{m}$ are the natural parameters. In the statistical literature this is known as multiple logistic regression.

\subsection{Parameter estimation}

The obvious starting point for training these models is to use maximum likelihood. For linear regression, this is equivalent to minimising the quadratic form

$$
(\mathbf{Y}-\mathbf{X} \boldsymbol{\beta})^{T}(\mathbf{Y}-\mathbf{X} \boldsymbol{\beta})
$$

with respect to beta, where $\mathbf{X}$ is the (input) data matrix and $\mathbf{Y}$ is the target matrix. Equating the derivative to zero yields the normal equations

$$
\left(\mathbf{X}^{T} \mathbf{X}\right) \boldsymbol{\beta}=\mathbf{X}^{T} \mathbf{Y},
$$

which can be solved efficiently by computing the pseudo-inverse $\mathbf{X}^{\dagger}$ of $\mathbf{X}$ and setting $\boldsymbol{\beta}=\mathbf{X}^{\dagger} \mathbf{Y}$. This is numerically more stable than computing explicitly the inverse of the square matrix $\mathbf{X}^{T} \mathbf{X}$.

The error function derived from maximum likelihood for both generalised linear models we are considering is not a quadratic form, and there is no direct solution using linear algebra; iterative methods are used instead. In principle, there is no reason why general purpose nonlinear optimisation algorithms should not be used, but it is more efficient to take advantage of the special "near-linear" form of the model. Let $\mathcal{L}$ denote the log likelihood of the observed variables,

$$
\mathcal{L}=P(\mathbf{Y} \mid \mathbf{X}, \boldsymbol{\beta})=\prod_{n=1}^{N} P\left(\mathbf{y}^{(n)} \mid \mathbf{x}^{(n)}, \boldsymbol{\beta}\right),
$$


making the usual assumption that the observations are independent and identically distributed. Let $\mathbf{H}=$ $\left(\partial^{2} \mathcal{L} / \partial \boldsymbol{\beta} \partial \boldsymbol{\beta}^{T}\right)$ denote the Hessian of $\mathcal{L}$. The Fisher scoring method is an iterative algorithm for determining the parameter estimates $\boldsymbol{\beta}$; at the $r$ th step the update formula is

$$
\boldsymbol{\beta}_{r+1}=\boldsymbol{\beta}_{r}-\{E[\mathbf{H}]\}^{-1} \frac{\partial \mathcal{L}}{\partial \boldsymbol{\beta}_{r}} .
$$

This is the same as the Newton-Raphson algorithm, except that the expected value of the Hessian replaces the Hessian. ${ }^{a}$ Normally taking a full Newton step in nonlinear optimisation is not a good idea, since it is easy to overshoot the optimum. However, there are two special features of the generalised linear model that make this procedure work well in practice: the log likelihood of logistic models has a single maximum, and it is possible to initialise the parameter vector $\boldsymbol{\beta}$ reasonably close to the maximum.

The Hessian of the log likelihood for the logistic model is equal to $-\mathbf{X}^{T} \mathbf{W X}$, where $\mathbf{W}$ is a diagonal weight matrix whose elements are $\pi^{(n)}\left(1-\pi^{(n)}\right)$ (where $\pi$ is defined just after (4)). The gradient of the $\log$ likelihood is equal to $\mathbf{X}^{T} \mathbf{W e}$, where the $n$th row of $\mathbf{e}$ is given by

$$
e^{(n)}=\left(y^{(n)}-\pi^{(n)}\right) / f^{\prime}\left(\eta^{(n)}\right) .
$$

We form the variable $\mathbf{z}_{r}=\mathbf{X} \boldsymbol{\beta}_{r}+\mathbf{e}$, which is the linearisation of the link function around the current value of the mean. Then (10) reduces to:

$$
\left(\mathbf{X}^{T} \mathbf{W}_{r} \mathbf{X}\right) \boldsymbol{\beta}_{r+1}=\mathbf{X}^{T} \mathbf{W}_{r} \mathbf{z}_{r}
$$

which is the normal form equation for a least squares problem with input matrix $\mathbf{X}^{T} \mathbf{W}_{r}^{1 / 2}$ and dependent variables $\mathbf{W}_{r}^{1 / 2} \mathbf{z}_{r}$ (compare with (8). The weight matrix $\mathbf{W}$ changes at each iteration, since it is a function of the parameter vector at the $r$ th step $\boldsymbol{\beta}_{r}$. The algorithm is known as Iterated Re-weighted Least Squares (IRLS); see McCullagh and Nelder. ${ }^{2}$

The reduction of the Newton step to the normal form Eq. (12) depends on being able to find a square root of $\mathbf{W}$ (which is easy in this case, as it is non-negative diagonal), and to compute $\mathbf{X}^{T} \mathbf{W}^{1 / 2}$ efficiently (which can be done without a full matrix multiplication as $\mathbf{W}$ is diagonal). We initialise the procedure by using the values $\left(y^{(n)}+0.5\right) / 2.0$ as a first estimate for $\pi^{(n)}$ and from this deriving the other quantities needed. The uniqueness of the maximum of $\mathcal{L}$ was shown in Auer et al. ${ }^{3}$

The case of multiple logistic or softmax regression is a little more complicated (and not so well documented in the literature). The gradient and Hessian of the $\log$ likelihood of a single input pattern $\mathbf{x}$ are given by

$$
\begin{gathered}
\frac{\partial \mathcal{L}}{\partial \beta_{k i}}=\left(p_{k}-y_{k}\right) x_{i} \\
\frac{\partial^{2} \mathcal{L}}{\partial \beta_{k i} \partial \beta_{l j}}=\left(p_{l} \delta_{k l}-p_{l} p_{k}\right) x_{i} x_{j},
\end{gathered}
$$

where $\delta_{k l}$ is the Kronecker delta. To show that there is a unique maximum, it is sufficient to prove that the Hessian $\mathbf{H}$ is positive semi-definite. If $\mathbf{a}$ is an arbitrary vector, and we write $\mathbf{C}=\left(p_{l} \delta_{k l}-p_{l} p_{k}\right)$, then

$$
\mathbf{a}^{T} \mathbf{H a}=\mathbf{a}^{T} \mathbf{x} \mathbf{C} \mathbf{x}^{T} \mathbf{a}=\left(\mathbf{x}^{T} \mathbf{a}\right)^{T} \mathbf{C}\left(\mathbf{x}^{T} \mathbf{a}\right) \geq 0
$$

since $\mathbf{C}$ is the covariance matrix of the multinomial distribution and is therefore positive semi-definite ${ }^{\mathrm{b}}$.

The next step in developing an algorithm similar to that used for a logistic model is to write the Hessian in the form $\boldsymbol{\Xi}^{T} \mathbf{W} \boldsymbol{\Xi}$. However, when we do this $\boldsymbol{\Xi}$ is a $(m n) \times(m p)$ block matrix containing $m$ copies of $\mathbf{X}$ along the diagonal (and $p$ is the input dimension), and the matrix $\mathbf{W}$ is an $m \times m$ block matrix, where each block is an $n \times n$ diagonal matrix containing the corresponding entries from $\mathbf{C}$ for each input pattern. (See Williams and Barber ${ }^{4}$ for details). Thus, unlike the case of the logistic model, $\mathbf{W}$ is not diagonal. To compute the square root of $\mathbf{W}$, we need only find a Cholesky decomposition of $\mathbf{C}$. However, because $\mathbf{C}$ has $m^{2}$ non-zero entries, it is no longer clear that this representation of the problem offers any practical advantage (in terms of efficiency) and so we have not taken this approach.

Instead, we have chosen to implement two alternative algorithms. In the first we calculate the exact Hessian by summing terms given by Eq. (13) for each row in the dataset. The resulting matrix is usually very ill-conditioned, but using singular value decomposition, it is numerically tractable to solve the original Fisher scoring equation (10). Alternatively, in a simplified algorithm, we treat each output as independent, which yields the same update rule as for the logistic model (this is no surprise, since the marginal

\footnotetext{
${ }^{\mathrm{a}}$ In any case, for the canonical link, the Hessian coincides with its expected value.

${ }^{b}$ Thanks to Chris Williams for pointing this out.
} 
distribution for a single output in a multiple logistic model is binomial with probability $p_{i}$ ), although this is not a good approximation to the true Hessian as it ignores all off-diagonal terms.

In either case, we initialise the parameters using the same procedure as for logistic regression, but treating each output independently. For convenience, we shall refer to these algorithms as IRLS, though strictly speaking the "weight" matrix approach is not used.

\section{Nonlinear RBF Networks for Classification}

The output of RBF networks is usually given as a linear combination of basis functions:

$$
o_{k}(x)=\sum_{j} \phi_{j}\left(\left\|\mathbf{x}-\mathbf{x}^{(j)}\right\|\right) w_{j k},
$$

where $\mathbf{x}^{(j)}$ is the "centre" of the $j$ th basis function. Once the parameters of the basis functions are fixed, the computation of the output weights is a linear regression problem, $\mathbf{Y}=\boldsymbol{\Phi} \mathbf{W}$, with $\boldsymbol{\Phi}$ denoting the design matrix, and $\mathbf{W}$ the output layer weights. (These are completely unrelated to the weights in Eq. (12). As in Eq. (7), this is solved by computing the pseudo-inverse of $\boldsymbol{\Phi}$. Because of the form of the solution, any linear constraint on the training targets is necessarily satisfied by the network outputs. ${ }^{5}$ For a multi-class classification problem, where a 1-of$m$ encoding is used, the network outputs will sum to one just as the targets do. However, the network outputs need not lie in the range $[0,1]$ and so it may not always be possible to interpret them as probabilities.

Instead, we can replace the linear output layer with logistic (for two-class) and softmax (for more than 2 classes) models. The parameters of the basis functions can be learned from the training data, as it is best to choose $\boldsymbol{\Phi}$ to approximate the input data density. ${ }^{1}$ We can then use the appropriate Fisher scoring algorithm from Sec. 2.2 to estimate the output layer weights W. Software implementing this model was developed using NetLAB, ${ }^{6}$ a neural network toolbox written in MATLAB ${ }^{\mathrm{c}}$.

We note that this approach can be used for any model which applies a logistic or softmax output activation to a function which is linear in its parame- ters, provided that there is a way of determining the matrix $\boldsymbol{\Phi}$.

\section{Experimental Results}

In this section we present the results of using our method of training logistic output RBF networks on classification problems. Their performance is compared with linear output models, and the training algorithm with scaled conjugate gradient (SCG) and quasi-Newton optimisation algorithms $\left(\right.$ Bishop $^{1}$ is a useful reference). The RBF networks used thin plate splines as basis functions (for the reasons given in Lowe $^{7}$ ). The centres were determined using either $K$-means or the EM algorithm (so that they approximate the unconditional density of the input data).

The initial output layer weights for the logistic models trained by SCG and quasi-Newton algorithms are taken from a linear output model trained with a pseudo-inverse, which is an efficient way to get a much better than random start point. The initialisation of the output weights for IRLS training was discussed in Sec. 2.2 and applies IRLS to a "smoothed" version of the targets.

Note that in all the results reported here, the reported computational effort does not include the centre selection phase and is solely for the training of the output layer weights and biases. All algorithms had the same stopping criterion; both the absolute change in the weight vector and the error function should be less than $1 \times 10^{-4}$.

\subsection{Synthetic datasets}

Two simple synthetic datasets have been created with a two-dimensional input space. In the two class case, data is drawn from a mixture of three Gaussians, two of which are assigned to one class. The generating parameters were selected so that the decision boundaries are non-linear.

The graph in Fig. 1 shows that with linear outputs, there are regions of the input space where the outputs are not confined to the interval $[0,1]$, and that this can occur even for training data. The learning curves in Fig. 2 show that the IRLS training algorithm is significantly faster than either SCG or quasi-Newton; this result is confirmed in Table 1.

\footnotetext{
${ }^{\mathrm{c}}$ Available from http://www.ncrg.aston.ac.uk/netlab/index.html The software used in this paper is available from
} the "Contributions" section. 


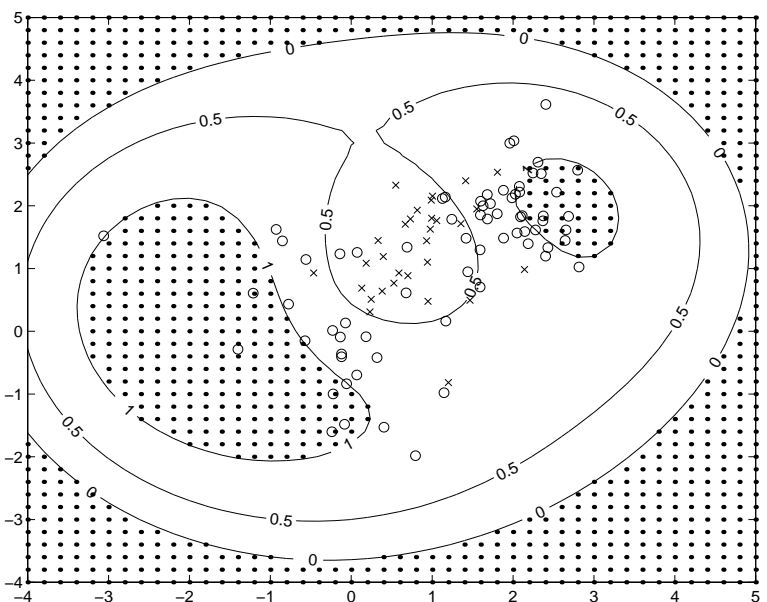

(a)

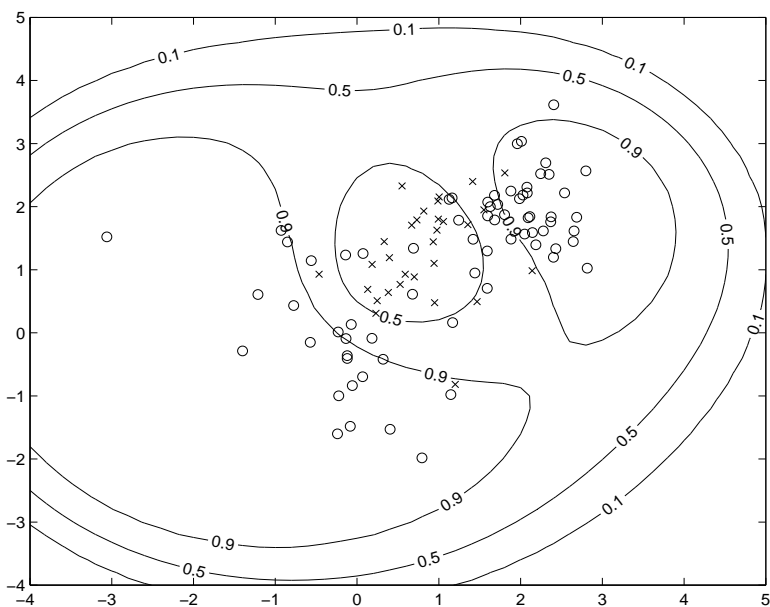

(b)

Fig. 1. Two-class synthetic data. (a) Contour plot of linear output RBF. Contours at 0, 0.5 and 1.0. In the shaded region the output cannot be interpreted as a probability. (b) Contour plot of logistic output RBF.

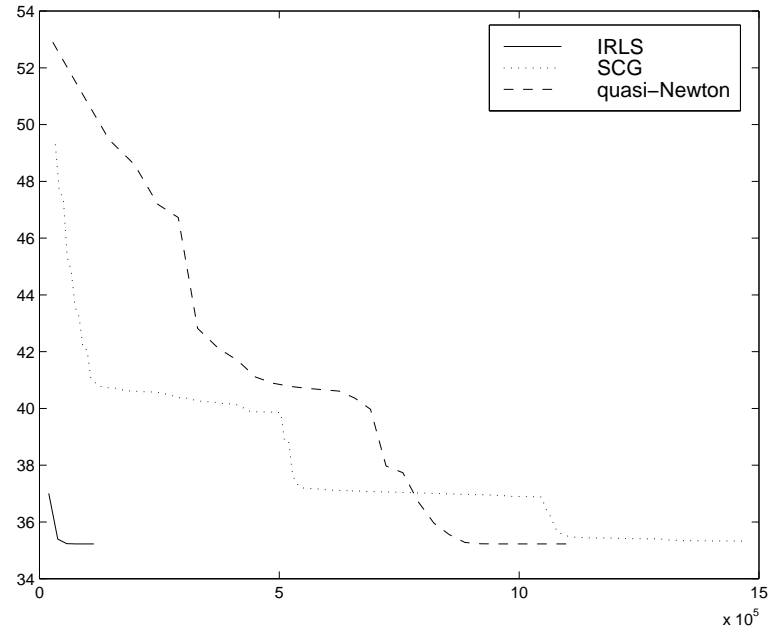

Fig. 2. Learning curves for logistic output RBF on two-class synthetic data. Negative log likelihood ( $y$-axis) against flops $\times 10^{5}$ ( $x$-axis).

To test the generality of this result, 10 replicated datasets were created by randomly sampling from the same mixture model. Table 2 contains the results of training 10 networks on these datasets. The error ratio is computed for each training set by dividing the test set error (i.e., negative log likelihood) of each algorithm by the minimum error across all the algorithms.

Table 2 shows that the IRLS is on average 6 times faster than the other two algorithms, and the computational effort is more consistent. It also finds
Table 1. Results on a two-class synthetic dataset.

\begin{tabular}{lrc}
\hline \multicolumn{1}{c}{ Algorithm } & \multicolumn{1}{c}{ Flops } & Error \\
\hline Linear outputs & 22323 & - \\
IRLS & 122087 & 35.2262 \\
SCG & 1467342 & 35.3275 \\
quasi-Newton & 1142511 & 35.2262 \\
\hline
\end{tabular}

Table 2. Results on replicated two-class synthetic dataset.

\begin{tabular}{llll}
\hline & IRLS & SCG & q-N \\
\hline Mean flops $\left(\times 10^{6}\right)$ & 0.1635 & 1.0036 & 1.0623 \\
S.d. flops $\left(\times 10^{5}\right)$ & 0.2137 & 4.0293 & 1.1322 \\
Mean error ratio & 1.000 & 1.0042 & 1.000 \\
S.d. error ratio & 0.000 & 0.0028 & 0.000 \\
\hline
\end{tabular}

the minimum error consistently, as does the quasiNewton algorithm, while scaled conjugate gradient usually converges slightly further away from the minimum and is slightly less consistent. The results for quasi-Newton and scaled conjugate gradient are in line with those achieved on other neural network training problems. ${ }^{6}$

The three-class synthetic dataset is drawn from a mixture of five Gaussians. Again, a linear output 


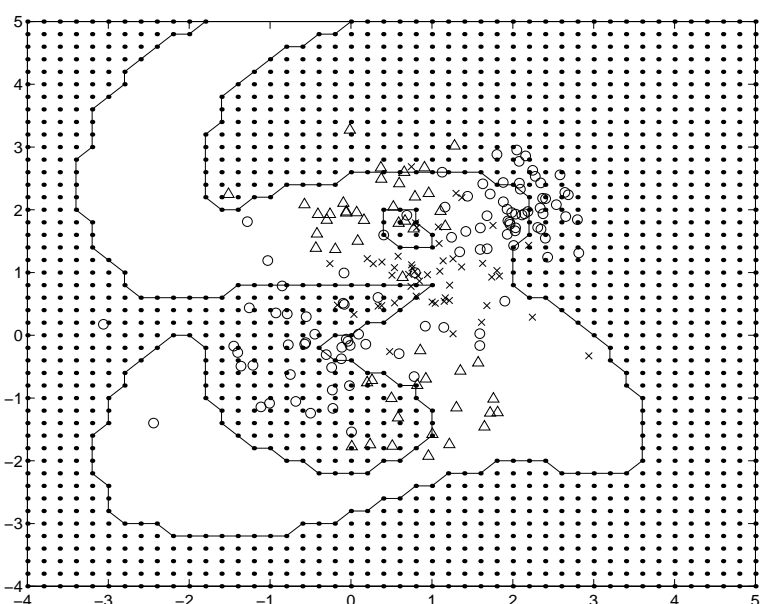

(a)

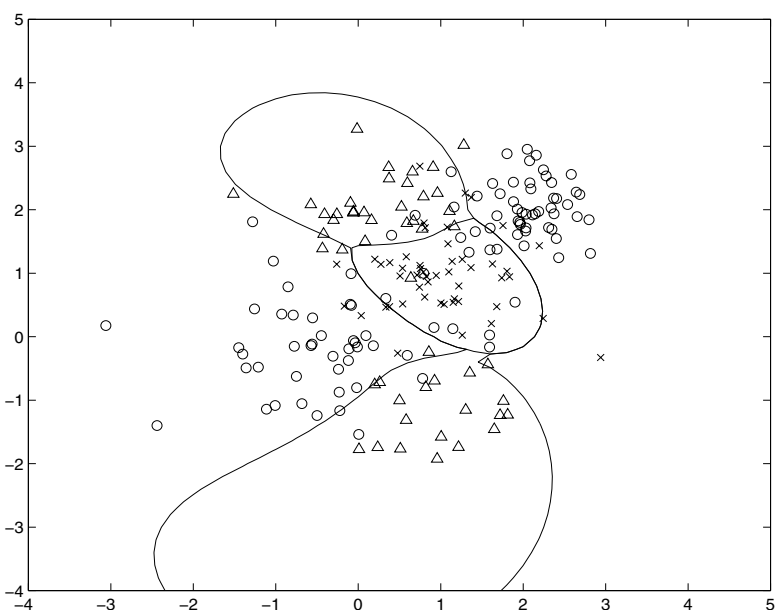

(b)

Fig. 3. Three-class synthetic data. (a) Non-probabilistic predictions from linear output RBF (dotted region). (b) Decision boundaries of softmax output RBF.

Table 3. Results on three-class synthetic data.

\begin{tabular}{lrc}
\hline \multicolumn{1}{c}{ Algorithm } & \multicolumn{1}{c}{ Flops } & Error \\
\hline Linear outputs & 59687 & - \\
Softmax: exact Hessian & 2050376 & 94.95 \\
Softmax: simplified & 478152 & 95.60 \\
SCG & 11031430 & 95.01 \\
quasi-Newton & 11370906 & 94.95 \\
\hline
\end{tabular}

Table 4. Results on replicated three-class synthetic dataset.

\begin{tabular}{llll}
\hline & $\begin{array}{l}\text { Softmax } \\
\text { exact } H\end{array}$ & SCG & q-N \\
\hline Mean flops $\left(\times 10^{7}\right)$ & 0.2271 & 1.0586 & 1.2256 \\
S.d. flops $\left(\times 10^{6}\right)$ & 0.3024 & 3.0739 & 0.6538 \\
Mean error ratio & 1.000 & 1.0042 & 1.000 \\
S.d. error ratio & 0.000 & 0.0013 & 0.000 \\
\hline
\end{tabular}

$\mathrm{RBF}$ can have non-probabilistic outputs in regions of input space where the density of training data is high (see Fig. 3a).

The specialised training algorithms were an order of magnitude more efficient than SCG and quasiNewton (see Table 3). The "simplified" algorithm was fastest to converge, but tends to take a large up-hill step when close to the maximum likelihood,

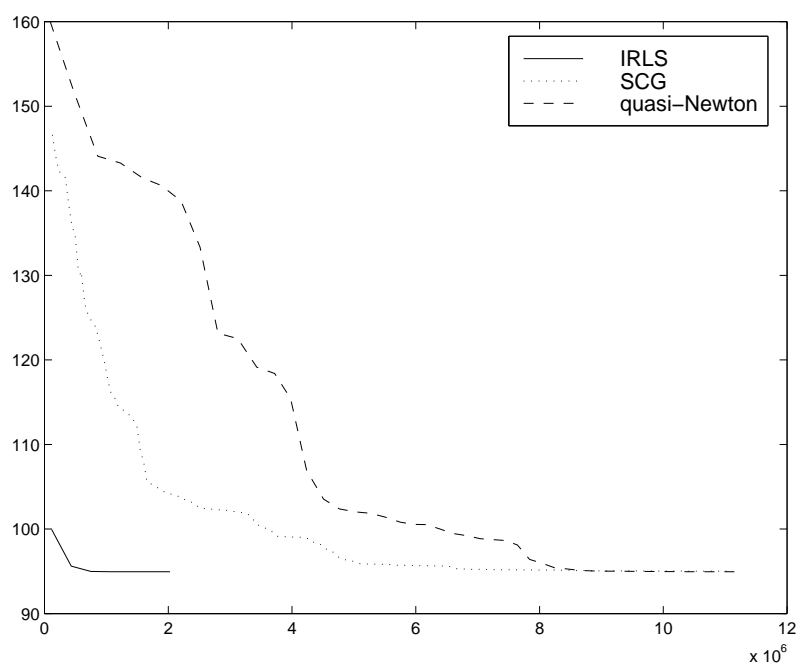

Fig. 4. Learning curves for logistic output RBF on three-class synthetic data. Negative log likelihood ( $y$ axis) against flops $\times 10^{6}$ ( $x$-axis).

so that the algorithm terminates at a sub-optimal value.

The results from 10 replicas of the three-class data are given in Table 4. The IRLS algorithm is on average more than 4.6 times faster than SCG, and the computational effort is again more consistent.a As for the two-class problem, the IRLS algorithm found the minimum reliably. For 7 of the 10 datasets, the approximate Hessian failed to improve on the initial weights, and so the results are not tabulated. 
Table 5. Results on crab data.

\begin{tabular}{lcc}
\hline \multicolumn{1}{c}{ Algorithm } & Flops & $\begin{array}{c}\text { Test Set } \\
\text { Misclassifications }\end{array}$ \\
\hline RBF: Linear outputs & 48733 & 6 \\
RBF: IRLS & 364836 & 4 \\
SCG & 3330815 & 4 \\
quasi-Newton & 3245640 & 4 \\
MLP & - & 3 \\
Linear Discriminant & - & 8 \\
Logistic Regression & - & 4 \\
Gaussian Process & - & 3 \\
MARS & - & 8 \\
\hline
\end{tabular}

\subsection{Real datasets}

We have tried out our method on three well-known classification problems ${ }^{\mathrm{d}}$. Leptograpsus crabs, diabetes in Pima women and forensic glass.

In the Leptograpsus crabs problem, the task is to determine the sex of crabs on the basis of 6 measurements. Using the same procedure reported in Ripley, ${ }^{8}$ we took 80 training examples and 120 test examples. The results, with some selected comparisons from Barber and Williams, ${ }^{9}$ are reported in Table 5. Note that SCG remained stuck in a local minimum despite several restarts, while IRLS and quasi-Newton achieved a similar much lower training set error value. Ten hidden units were used, since this was the smallest number for which the logistic $\mathrm{RBF}$ trained to a sufficiently low error.

In the diabetes diagnosis problem, the task is to diagnose whether a subject has diabetes or not on the basis of 8 variables measuring various disease indicators. There are 200 training examples, and 332 test examples. The default classifier (assigining every subject to the healthy class) has an error rate of $33 \%$. The optimal RBF network (chosen using crossvalidation) had 8 hidden units: its results are compared with those achieved by other models (as given in Ripley ${ }^{10}$ ) in Table 6 .

In the forensic glass problem, the task is to determine the type of a glass sample from the refractive index and composition (weight fraction of eight oxides). There are 214 examples with 6 classes, so performance is estimated using 10-fold crossvalidation. ${ }^{10}$ To improve performance, a committee of 10 networks was used for each partition, as was
Table 6. Results on diabetes data.

\begin{tabular}{lrc}
\hline \multicolumn{1}{c}{ Algorithm } & \multicolumn{1}{c}{ Flops } & $\begin{array}{c}\text { Miclassification } \\
\text { Rate } \%\end{array}$ \\
\hline RBF: Linear outputs & 96723 & 19.9 \\
RBF: IRLS & 436145 & 21.4 \\
SCG & 1469126 & 21.4 \\
quasi-Newton & 6493136 & 21.4 \\
MLP & - & 22.6 \\
Linear Discriminant & - & 20.2 \\
Logistic Regression & - & 19.9 \\
Gaussian Process & - & 20.5 \\
MARS & - & 22.6 \\
\hline
\end{tabular}

Table 7. Results on forensic glass data. The simplified IRLS algorithm failed to converge.

\begin{tabular}{lc}
\hline \multicolumn{1}{c}{ Algorithm } & Misclassification Rate \% \\
\hline RBF: Linear outputs & 31.4 \\
RBF: IRLS & 30.3 \\
MLP & 23.8 \\
Linear Discriminant & 36.0 \\
Gaussian Process & 23.3 \\
MARS & 32.2 \\
\hline
\end{tabular}

done by Ripley ${ }^{10}$ for the MLP. The results are contained in Table 7; for comparison, the default rule (assigning to the largest class) has a misclassification rate of $65 \%$.

It should be noted that the computational effort for both MLP and Gaussian Process methods on this problem was very large (the latter required 24 hours on an SGI Challenge) compared with the softmax RBF approach (which took about 20 minutes on a less powerful computer). On the third dataset, the optimal number of hidden units for the linear output RBF was 25 , while it was 12 for the non-linear output RBF.

\section{Discussion and Conclusions}

In this paper we have demonstrated that the benefits of using non-linear output functions for classification problems can be achieved with RBF networks while still retaining their significant training speed advantage over MLPs. This approach can be applied to any

$\overline{{ }^{d} \text { Available from http://markov.stats.ox.ac.uk/pub/PRNN }}$ 
generalised linear regression model where the nonlinear function parameters can be estimated directly from the input training data.

The IRLS algorithm is considerably faster than using more general non-linear optimisation methods. In our experiments, IRLS achieved the same final error values as the quasi-Newton algorithm (to 4 decimal places), while scaled conjugate gradient often terminated at error values that were larger in the third significant figure. A simplified, more efficient, algorithm for multi-class problems, which approximates the Hessian of the log likelihood, did not converge in sufficiently many cases to be of practical value.

In the future we hope to extend many useful results for RBFs that depend on the pseudo-inverse solution for the output weights to the non-linear output models considered in this paper using Eq. (12). For example, Lowe ${ }^{11}$ explains the link between the degrees of freedom of an RBF model and the eigenvalues of the design matrix and Webb and Lowe ${ }^{12}$ give an interpretation of the hidden units. The single maximum of the log likelihood means that a Bayesian approach to regularisation with the Laplace approximation is likely to be effective and we intend to pursue this further. In Hastie and Tibsharani ${ }^{13}$ there is an explanation of how to calculate the degrees of freedom for a generalised additive model, and it should be possible to apply this to RBFs.

\section{Acknowledgments}

This paper has benefitted from discussions with David Lowe and Chris Williams.

\section{References}

1. C. M. Bishop, Neural Networks for Pattern Recognition (Oxford University Press, 1995).

2. P. McCullagh and J. A. Nelder, Generalized Linear Models (Chapman and Hall, London, 1983).

3. P. Auer, M. Herbster and M. K. Warmuth, Exponentially many local minima for single neurons, in Neural Information Processing Systems 8, (1996), pp. 316-322.

4. C. K. I. Williams and D. Barber, Bayesian classification with Gaussian Processes, IEEE Transactions on Pattern Analysis and Machine Intelligence, 20 (1998), 1342-1351.

5. D. Lowe and A. R. Webb, Optimized feature extraction and the Bayes decision in feed-forward classifier networks, IEEE Transactions on Pattern Analysis and Machine Intelligence 4 (1991), 355-364, .

6. I. T. Nabney, Netlab: Algorithms for Pattern Recognition (Springer, 2002).

7. D. Lowe, On the use of nonlocal and non positive definite basis functions in radial basis function networks in IEE ANN 1995, (1995), p. 206-211.

8. B. D. Ripley, Flexible non-linear approaches to classification, in From Statistics to Neural Networks, eds. V. Cherkassy, J. H. Friedman, and H. Wechsler (Springer, 1994), p. 105-126.

9. D. Barber and C. K. I. Williams, Gaussian processes for Bayesian classification via hybrid Monte Carlo, in Neural Information Processing Systems 9 eds. M. C. Mozer, M. I. Jordan, and T. Petsche, (1997).

10. B. D. Ripley, Pattern Recognition and Neural Networks (Cambridge University Press, 1996).

11. D. Lowe, Characterising complexity in a radial basis function network, in IEE ANN 1997 (1997), p. $19-23$.

12. A. R. Webb and D. Lowe, The optimised internal representation of multilayer classifier networks performs nonlinear discriminant analysis, Neural Networks 3 (1990), 367-375.

13. T. J. Hastie and R. J. Tibsharani, Generalized Additive Models (Chapman and Hall, London, 1990). 